\title{
Nouveaux regards sur la prééclampsie
}

Céline Méhats, Francisco Miralles, Daniel Vaiman

> La prééclampsie est une maladie majeure, fréquente et potentiellement sévère de la grossesse, caractérisée par une hypertension gravidique et une protéinurie. Dans cette revue, nous rapportons les progrès récents réalisés dans la compréhension de la pathologie et évoquons ses impacts à long terme sur la santé vasculaire maternelle. Nous décrivons ensuite les bases génétiques, épigénétiques et immunologiques de la prééclampsie. Nous détaillons en particulier les liens entre pathologie et stress oxydatif au niveau des cellules placentaires (trophoblastes) et endothéliales. Nous mentionnons les modèles d'étude cellulaires et animaux couramment utilisés pour décrypter les voies physiopathologiques modifiées dans une grossesse prééclamptique par rapport à une grossesse normale. Enfin, nous évoquons les possibilités thérapeutiques existantes et à venir pour améliorer le suivi des grossesses, la santé des patientes et celles des enfants nés de grossesses prééclamptiques. <

\section{La prééclampsie}

Une obsession angoissée des services de gynécoobstétrique dans les pays industrialisés

Une jeune mère consulte pour une grossesse «compliquée »: œdèmes des membres inférieurs, tension artérielle très élevée; des mots savants sont utilisés par les médecins discutant devant son lit, sans qu'elle en comprenne le sens: «protéinurie, labétalol, etc. ». Puis le bébé naît prématuré à 36 semaines d'aménorrhée (huit mois de grossesse) avec un poids inférieur au poids attendu à cet âge gestationnel. Au cours de sa présence à l'hôpital, la jeune mère n'a pas entendu le mot «prééclampsie», ce qui, plus tard, va compliquer ses recherches pour essayer de mieux comprendre ce qui lui est arrivé. Puis le temps va passer, le bébé va grandir et les «problèmes » seront oubliés.

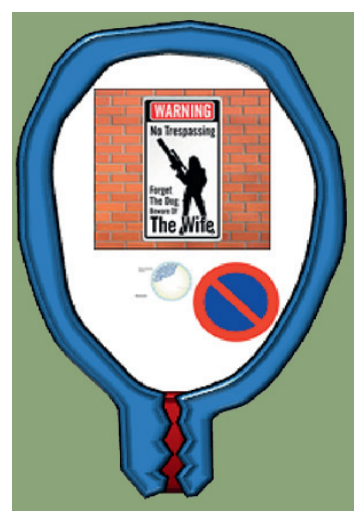

Inserm U1016, CNRS UMR8104, Institut Cochin, équipe FGTB, 24, rue du faubourg SaintJacques, 75014 Paris, France. daniel.vaiman@inserm.fr

La prééclampsie $(P \varepsilon)$, longtemps considérée comme une maladie mystérieuse à l'étiologie inconnue, naguère dénommée toxémie gravidique, se caractérise par une hypertension artérielle et une protéinurie se déclenchant au plus tôt à la mi-gestation [1] $(\rightarrow)$.

La maladie induit des répercussions cliniques parfois gravissimes, incluant le syndrome HELLP (hemolysis, elevated liver enzymes, low platelet), pouvant se compliquer d'une coagulation intra- $(\rightarrow)$ Voir la Nouvelle de V. Rigourd et al., $\mathrm{m} / \mathrm{s} \mathrm{n}^{\circ} 12$, décembre 2008, page 1017 vasculaire disséminée, ou par l'éclampsie, une crise convulsive généralisée décrite dès l'antiquité, avec un premier cas peut-être identifié il y a 28000 ans [2].

Au cours des 15 dernières années, des progrès considérables ont été réalisés dans la compréhension de l'étiologie de la PE. La pathologie est considérée comporter deux phases: I'une asymptomatique et très précoce, en relation avec une placentation défectueuse; l'autre caractérisée par l'apparition des symptômes chez la mère.

\section{De la dysfonction placentaire à l'attaque}

du système vasculaire maternel :

des conséquences maternelles et fœtales parfois dramatiques La première phase de la $P E$ se caractérise par un défaut d'invasion des trophoblastes qui colonisent les tissus utérins maternels, induisant des anomalies histologiques locales. La deuxième phase correspond au syndrome maternel affectant l'endothélium, probablement par la libération, par le placenta, de molécules anti-angiogéniques dans la circulation maternelle comme sFLTl (soluble fms-like tyrosine kinase-1) et $s E N G$ (soluble endoglin) (Figure 1). sFLTl (ou sVEGFRI), la forme soluble du récepteur du VEGF (vascular endothelial growth factor) générée par épissage alternatif, inhibe la formation des tubes endothéliaux et bloque les effets vasodilatateurs de ses ligands (VEGF et de PIGF). Similairement, la sENG (I'endogline soluble) joue un rôle anti-angiogénique en inhibant la cascade du TGF $\beta$ (transforming growth factor $\beta$ ), cruciale pour l'angiogenèse. 


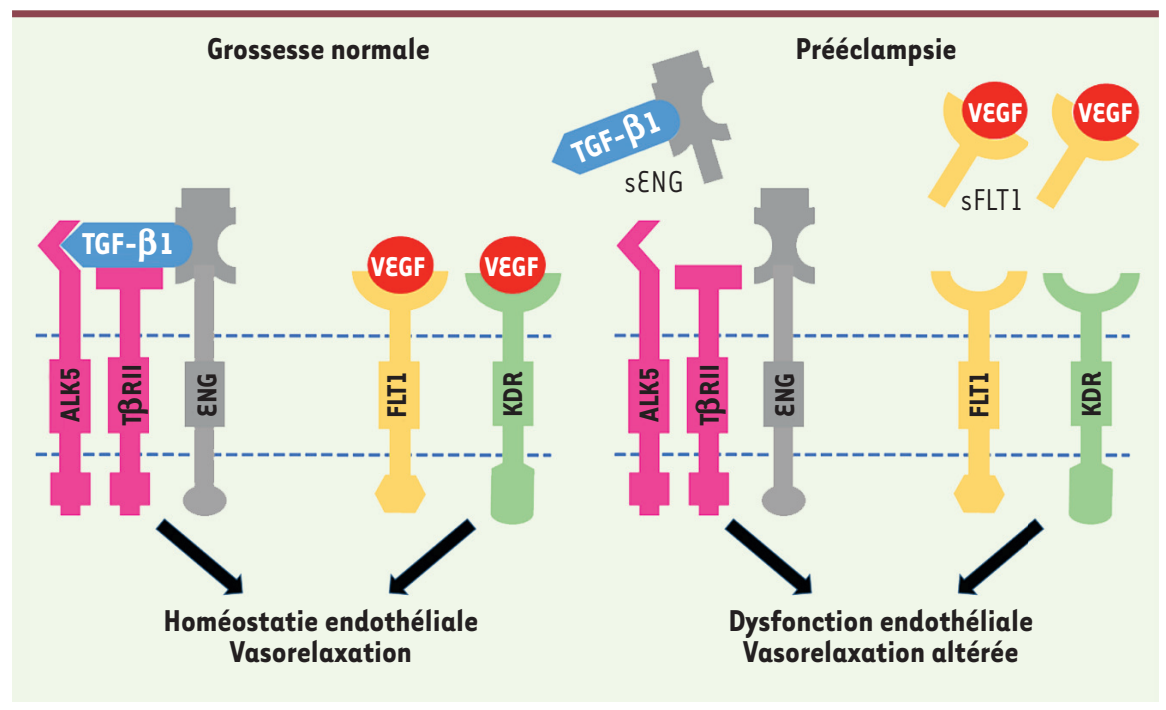

Figure 1. Dans la prééclampsie, on observe une invasion insuffisante des artères spiralées utérines. Cette constatation anatomique n'explique pas la dysfonction endothéliale généralisée retrouvée chez les patientes. Dans leur plasma, des facteurs circulants anti-angiogéniques se trouvent augmentés au plus tôt à la $20^{e}$ semaine d'aménorrhée. Les principaux facteurs identifiés sont des récepteurs solubles, sENG et sFLTl, des facteurs pro-angiogéniques tels que certaines protéines de la famille du TGF $\beta$, le VEGF, ou le PIGF (placental growth factor) (non représenté sur la figure mais capable d'interagir avec le récepteur FLTl). L'excès de ces pièges à molécules angiogéniques accompagne la pathologie humaine. Chez l'animal, on peut mimer la PE en dérégulant le système sFLTl/VEGF/FLTl. La question de la causalité princeps de ce système dans la pathologie demeure cependant (figure adaptée de [9]). TGF- $\beta 1$ : transforming growth factor beta 1 ; ALK5 : activin receptor-like kinase 5 ; TRRII : TGF-beta type II receptor; ENG : endoglin; sENG : soluble endoglin; FLTl : fms related tyrosine kinase 1 ; sFLTl : soluble FLT1 ; KDR : kinase insert domain receptor; VEGF : vascular endothelial growth factor; PIGF : placental growth factor.

Le PIGF joue aussi un important rôle pro-angiogénique, et une quantité trop limitée de ce facteur au cours du premier trimestre de la grossesse contribue au risque de développer une PE. L'historique de la découverte de ces facteurs est détaillé dans une excellente revue de 2011 [3]. D'autres molécules peuvent contribuer à l'effet pro-angiogénique nécessaire à une grossesse normale, comme récemment documenté pour le système PROK (prokinéticines)/

$(\rightarrow)$ Voir la Synthèse de S. Brouillet et al., $m / s n^{\circ} 3$, mars 2014, page 274

PROKR $[4,47](\rightarrow)$.

L'atteinte vasculaire au cours de la grossesse, induirait l'ensemble des symptômes de la maladie. Un tiers des cas de PE s'accompagne d'un retard de croissance intra-utérin ( $R C I U$ ), marquant un défaut d'efficacité placentaire, hypothétiquement compensé dans un certain nombre de cas, par l'hypertension maternelle.

\section{La prééclampsie programme une fragilité}

\section{cardiovasculaire de long terme}

La grossesse peut être considérée comme une gageure pour l'organisme maternel, impliquant des modifications rapides, pondérales, vasculaires, immunitaires et endocrines. Ce défi, quand il se complique d'une $P \varepsilon$, peut laisser des traces. Les patientes prééclamptiques voient leur risque d'avoir besoin d'une greffe rénale multiplié par 5 [5]. La $P \varepsilon$ est aussi un facteur de risque cardiovasculaire pour la mère, mais également pour l'enfant exposé à un environnement utérin néfaste [6] susceptible d'altérer son système cardiovasculaire et ses reins de façon durable, en induisant des conséquences à long terme lorsqu'il sera adulte. Dans les cas de RCIU, une réduction du nombre de glomérules rénaux a été mise en évidence dans les modèles animaux [7], et les observations réalisées chez l'homme suggèrent, de même, une réduction de la taille des reins ainsi qu'une augmentation moyenne de la pression artérielle chez les enfants ayant vécu ce type de grossesse [8].

Sept années après avoir souffert d'une PE, $20 \%$ des femmes présentent une hypertension et une protéinurie (traduisant une altération rénale). Cette fréquence n'est que de $2 \%$ dans la population [9]. Les risques de pathologie cardiovasculaire chronique sont également augmentés et une diminution de l'espérance de vie est observée [10], en particulier dans les cas de $P \varepsilon$ précoce, avec une survie de $90 \%$ trente ans après la grossesse, et d'environ $98 \%$ dans les cas de PE plus tardives, contre une survie proche de $100 \%$ chez les contrôles (Figure 2). La compréhension des mécanismes impliqués est très limitée en raison, en particulier, de l'impossibilité de pouvoir les étudier chez l'homme. Une alternative serait l'utilisation de modèles animaux afin d'évaluer les conséquences cardio-vasculaires à long terme.

\section{Bases moléculaires de la prééclampsie}

La prééclampsie et les gènes

La PE est une pathologie complexe et polygénique dont la part génétique (c'est-à-dire l'héritabilité) a été estimée dans de grandes cohortes scandinaves. L'étude de Cnattingius et al., en 2004, a analysé l'occurrence de la PE chez 244564 femmes apparentées (couples de sœurs, couples mère-sœur, etc.) à l'origine de 701488 grossesses. Elle conclut à une héritabilité de plus de $50 \%$ que l'on peut décomposer en $35 \%$ liés à 


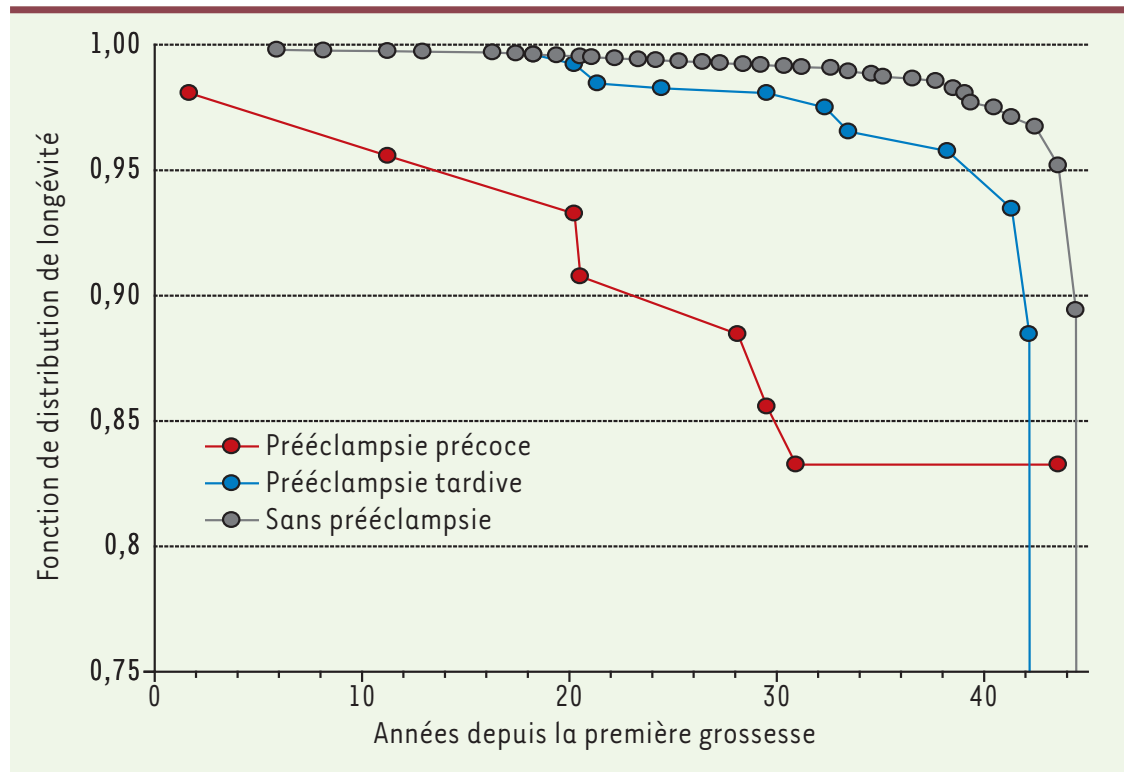

Figure 2. Courbes de survie des femmes ayant souffert ou non d'une prééclampsie. En moyenne, on estime que l'espérance de vie après une prééclampsie est diminuée de 7 ans. La question de savoir si cet état de fait est lié à une génétique cardiovasculaire moins performante ou causée par la pathologie reste ouverte (d'après [10]).

la génétique maternelle, $20 \%$ à la génétique fœtale, $13 \%$ à un effet «couple», moins de $1 \%$ à un effet environnemental partagé par les apparentées, et $32 \%$ à des facteurs inconnus [11]. Des variants génétiques de gènes cibles impactent également le risque de développer une $P E$ (pour une revue voir [12]), comme les gènes codant les facteurs 2 et 5 de la coagulation (F5, F2), l'angiotensinogène (AGT) et son récepteur (AGTRl), ou l'enzyme de conversion de l'angiotensinogène $(A C E)$. Des études familiales ont également permis de relier des régions génomiques au risque de $P \varepsilon$, avec l'identification de gènes comme STOXI (storkhead box 1) ${ }^{1}$, ou TNFSFI3B (TNF superfamily member $13 b)^{2}$. La première étude d'association GWAS (genome-wide association study) concernant la $P E$ a été publiée très récemment [13]. Cette analyse, réalisée sur 4380 cas et 310238 témoins, a révélé un variant significatif (rs4769613) localisé à proximité du gène FLTI, confirmant ainsi l'impact de ce gène, codant en particulier sFLTl, dans la pathologie. Ce résultat démontre aussi un probable «émiettement » de la génétique de la $P E$ sur de nombreux locus ayant, individuellement, un effet mineur dans la population générale, mais un effet majeur, dans des populations limitées, les rendant susceptibles.

\section{Prééclampsie et régulation épigénétique}

Trois types de machineries moléculaires épigénétiques coexistent dans la cellule : la méthylation de l'ADN, essentiellement au niveau des dinucléotides CG ; les modifications post-traductionnelles des histones; et la régulation des ARN non-codants, en particulier les microARN (miARN).

${ }^{1}$ STOXI code un facteur de transcription intervenant dans le contrôle de la prolifération et de l'invasion des trophoblastes.

${ }^{2}$ Ou BAFF (B-cell activating factor)
La méthylation de l'ADN a été particulièrement étudiée dans le contexte de la PE. Certains gènes sont en effet anormalement méthylés, comme SERPINA3 (serpin family a member 3) qui code un inhibiteur plasmatique de sérine protéases [14]. De nombreux articles se sont également intéressés aux rôles des miARN dans la PE [15]. Ces ARN courts noncodants exercent une série de fonctions cruciales pour la physiologie trophoblastique [16] et certains présentent des concentrations sériques anormales au cours de la PE. Leur potentiel pronostique a d'ailleurs été examiné dans une étude récente [17] dans laquelle les auteurs ont analysé 12 études cas-témoin. Ils ont conclu à une très grande hétérogénéité des résultats, que ce soit pour les taux de miARN mesurés dans le placenta ou ceux détectés dans le sérum. Un effort supplémentaire impliquant de grandes cohortes apparaît donc indispensable pour rendre robustes les données concernant les concentrations de miARN retrouvées dans le contexte d'une prédisposition à la $P \varepsilon$. Une autre importante marque épigénétique est constituée par l'association locale de variants d'histone spécifiques dans des régions chromatiniennes déterminées. Ce domaine demeure encore largement inexploré.

\section{Bases immunologiques de la prééclampsie}

La régulation immunologique est fondamentale au cours de la grossesse (Figure 3). Dans les pathologies placentaires, une gradation de la tolérance immunologique peut en effet être observée, depuis des cas de rejets particulièrement importants (fausses-couches à répétition), jusqu'à, à l'opposé, une identité moléculaire qui induit une pathologie de contact facilité (ou placenta percreta ${ }^{3}$ ). Le lien entre immunité et pathologie placentaire est le sujet d'une revue détaillée publiée par LaMarca et al. en 2013 [18]. Deux éléments qui révèlent un rôle de l'immunité dans la $P E$ sont soulevés dans cette revue : le premier est l'observation de la prévalence des cas de $P E$ chez des femmes primipares, ou lors d'un changement de paternité, lors d'une grossesse ultérieure. Il souligne un lien entre la PE et l'exposition au sperme, donc aux antigènes paternels [19]; le deuxième est la mise en évidence de l'importance de la

${ }^{3}$ Le placenta percreta se définit par une invasion placentaire étendue avec atteinte conjuguée du myomètre et de la séreuse utérine et par un envahissement plus ou moins important des organes pelviens adjacents. 


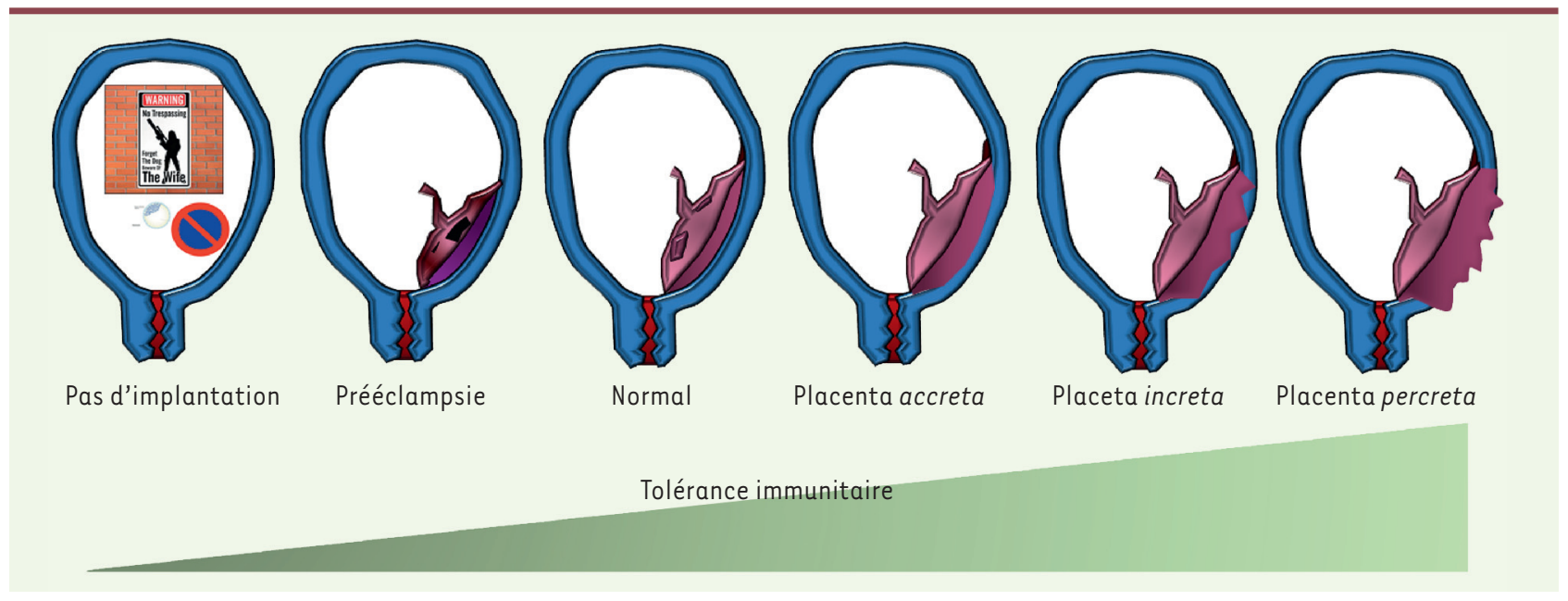

Figure 3. Il existe de forts arguments pour penser que la prééclampsie est avant tout une pathologie ayant une base immunologique. Les observations de Robillard [19] montrent que l'habituation à des antigènes paternels spermatiques induit une «éducation » du système immunitaire maternel, une meilleure implantation, et une meilleure placentation. La fréquence de la prééclampsie couramment estimée entre 2 et $8 \%$ selon les populations dépasse $30 \%$ dans les cas de primo-rapports non protégés fécondants (P.Y. Robillard, communication personnelle). Dans les cas de trop forte tolérance immunitaire, la régulation de l'invasion trophoblastique est défaillante et le placenta peut envahir la paroi utérine, voire la traverser (placenta percreta). Dans ces cas, il existe des risques accrus d'hémorragie de la délivrance, principale cause de mortalité maternelle dans les pays industrialisés.

combinaison des haplotypes de récepteurs KIR (killer cell immunoglobulin-like receptors), spécifiques des lymphocytes NK (natural killer), qui sont exprimés à la surface des cellules d'origine maternelle, et les allotypes HLA-C du complexe majeur d'histocompatibilité, qui sont présentés par les trophoblastes fœtaux [20]. En effet, la combinaison d'un génotype maternel AA de KIR, et d'un HLA-C2 fœtal, est associée à un risque accru de $P E$.

Les lymphocytes $T$ régulateurs (Treg) participent également à la régulation des interactions fœto-placentaires. Une étude récente a en effet comparé les différentes populations de leucocytes, sur des coupes paraffinées de placenta issus de 18 échantillons contrôles et de 11 échantillons de placenta increta/accreta ${ }^{4}$ [21]. Dans ces cas pathologiques associés à une pénétration trop profonde des trophoblastes dans les tissus maternels, le nombre de lymphocytes Treg est augmenté (multiplié par 6,8), celui des cellules dendritiques étant, quant à lui, diminué (divisé par 2,32). Dans son ensemble, le nombre de lymphocytes $\mathrm{CD} 4^{+}$est accru (multiplié par 3,38). Dans la PE, des tendances inverses sont observées pour les lymphocytes Treg, avec une diminution significative et des modifications, tout à fait similaires, dans les cas de fausse-couche à répétition, une pathologie qui touche 1 à $5 \%$ des grossesses.

\section{Prééclampsie et stress oxydatif Le stress oxydatif}

Le stress oxydatif (ou oxydant) est une agression des constituants de la cellule par des espèces réactives de l'oxygène ( $\varepsilon R O$ - ou ROS

${ }^{4}$ Dans le placenta accreta, les villosités sont en contact avec le myomètre et y pénètrent plus ou moins profondément; dans le placenta increta, les villosités envahissent le myomètre. en anglais - comme $\mathrm{O}_{2}^{--}, \mathrm{H}_{2} \mathrm{O}_{2}, \mathrm{HO}^{\circ}$ ) et aux espèces réactives de l'azote oxygéné (ERA - ou RNS - tels que $\mathrm{NO}^{*}$ et $\mathrm{ONOO}^{-}$) [22]. Alors que leur production est un phénomène physiologique, essentiel au métabolisme cellulaire, les $\varepsilon R O$ et les ERA peuvent être à l'origine de dommages structuraux et fonctionnels de l'ADN, de I'ARN, des protéines et des lipides des membranes cellulaires (Figure 4). Ils ont pour origine des mécanismes de stress oxydant qui sont générés dans la mitochondrie (chaîne respiratoire), le réticulum endoplasmique (mono-oxygénases), la membrane plasmatique (oxydases), les peroxysomes ou le cytoplasme.

\section{Stress oxydant et prééclampsie}

L'augmentation du stress oxydant dans la PE est bien documentée [23]. Dans le placenta prééclamptique, le défaut du remodelage des artères spiralées restreint l'afflux de sang maternel au fœtus. Cela produit de façon exagérée, par rapport à la normale, des fluctuations de la concentration d'oxygène à l'origine d'épisodes d'ischémie-reperfusion et de la production de ERO et d'ERA [24] $(\rightarrow)$.

Ces perturbations induisent une apoptose trophoblastique et la libération de débris cellulaires et de facteurs anti-angiogéniques

$(\rightarrow)$ Voir la Synthèse de J.C. Challier et S. Uzan, $m / s n^{\circ} 11$, novembre 2003, page 1111 dans la circulation maternelle, ce qui provoque, in fine, une inflammation et une dysfonction systémique de l'endothélium maternel. 


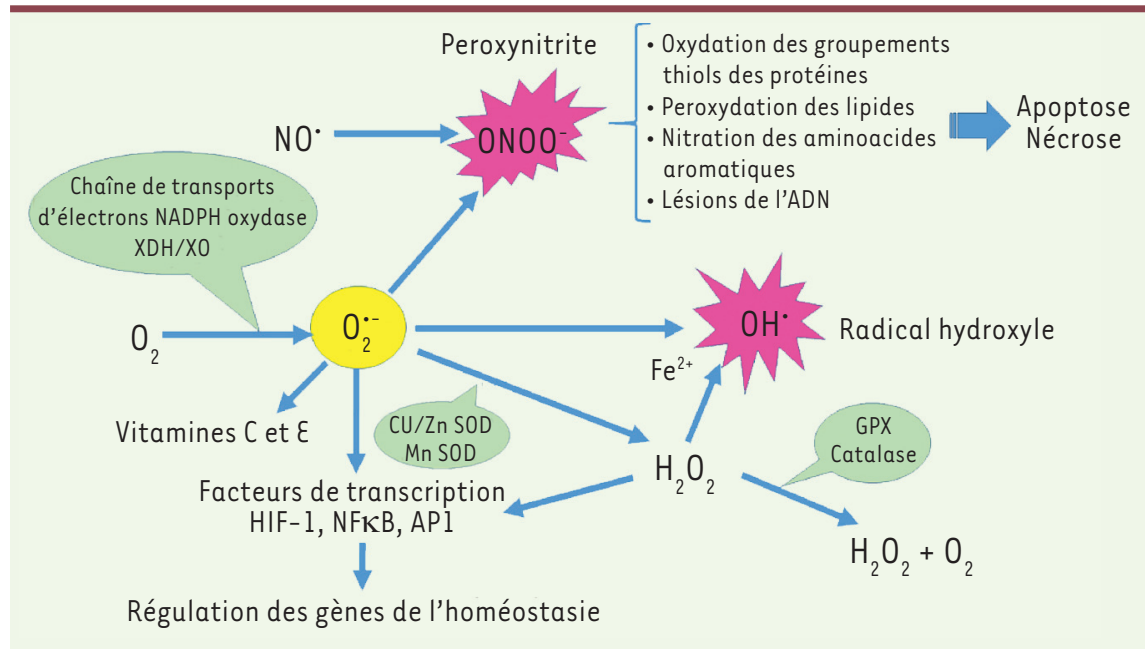

Figure 4. Les cascades du stress oxydatif jouent un rôle fondamental dans la pathologie prééclamptique. Les principales molécules et voies de détoxification sont présentées dans la figure (d'après [22]). $\mathrm{NO}^{\circ}$ : monoxyde d'azote; $\mathrm{ONOO}^{-}$: peroxynitrite ; $\mathrm{OH}^{*}$ : radical hydroxyle $; \mathrm{O}_{2}{ }^{--}$: radical superoxyde; $\mathrm{H}_{2} \mathrm{O}_{2}$ : peroxyde d'hydrogène ; NADPH : nicotinamide adénine dinucléotide phosphate oxydase; XDH : xanthine déshydrogénase; $X O$ : xanthine oxydase; GPX: glutathion peroxydase ; SOD : superoxyde dismutase; HIF-1 : hypoxia inducible factor 1 ; NFKB : nuclear factor kappa $B ; A P 1$ : activator protein 1 .

\section{Mécanismes de production du stress oxydant dans le placenta}

Une grande proportion des $\varepsilon R O$ produits dans le placenta prééclamptique pourrait provenir de l'activité mitochondriale, soit sous hypoxie, soit sous hyperoxie. Des analyses du protéome de placentas prééclamptiques ont en effet révélé des modifications en lien avec la chaîne respiratoire et la génération d'ERO, montrant l'importance des mitochondries dans le développement de la PE [25]. Des altérations du taux de consommation d'oxygène indiquent également une dysfonction mitochondriale dans les trophoblastes isolés de placentas prééclamptiques. La reperfusion placentaire provoque également des dommages oxydants par la conversion de la xanthine déshydrogénase (XD) en xanthine oxydase $(X O)$. En effet, la XO convertit la xanthine (ou l'hypoxanthine) et $\mathrm{I}^{\prime} \mathrm{O}_{2}$ en acide urique et en $\mathrm{H}_{2} \mathrm{O}_{2}$. Cette enzyme peut également produire du $\mathrm{O}_{2}{ }^{\cdot-}$. Dans le placenta, le fer stocké dans les syncytiotrophoblastes catalyse (par la réaction de Fenton) la conversion du $\mathrm{H}_{2} \mathrm{O}_{2}$ en $\mathrm{OH}^{*}$, un radical extrêmement réactif et cytotoxique, et plusieurs études ont montré que les trophoblastes isolés à partir de placentas prééclamptiques présentent une augmentation de l'activité de la X0 par rapport aux trophoblastes isolés de placentas non-pathologiques [26]. L'activité NADPH (nicotinamide adénine dinucléotide phosphate) oxydase (NOX) est également augmentée dans les placentas prééclamptiques. Les NOX forment des complexes enzymatiques liés aux membranes cellulaires. Ils catalysent la réduction de l' $\mathrm{O}_{2}$ en utilisant le NADPH ou le NADH (nicotinamide adénine dinucléotide) comme source d'électrons. Cette réaction est une source importante $\mathrm{d}^{\prime} \mathrm{O}_{2}{ }^{--}$. Les cellules endothéliales HUVEC (human umbilical vein endothelial cells) traitées par du sérum provenant de femmes prééclamptiques, surexpriment la sous-unité NADPH oxydase gp9l (phox). Cela conduit à la production de quantités élevées de $0_{2}{ }^{*-}$ [27]. La surexpression de NOX2 (NADPH oxidase 2) a également été montrée dans les cellules HUVEC de patientes prééclamptiques. L'activation des NOX est stimulée par la liaison de l'angiotensine II (AngII) au récepteur ATI-AA (Angll type I receptor). Le placenta produit alors des $\varepsilon R 0$ qui activent le facteur nucléaire kappa $B(N F-\kappa B)$, déclenchant ainsi une réponse inflammatoire [28].
L'anion $\mathrm{O}_{2}{ }^{--}$peut, à lui seul, produire des lésions tissulaires majeures dans le placenta. Sa réaction avec le NO conduit également à la formation de peroxynitrite $\left(\mathrm{ONOO}^{-}\right)$, un agent pro-oxydant très puissant. Cette réaction, très rapide, peut concurrencer la détoxification de $\mathrm{l}^{\prime} \mathrm{O}_{2}{ }^{--}$par les superoxyde dismutases (SOD). Le peroxynitrite réagit lentement, et sélectivement dans les systèmes biologiques, essentiellement sur les tyrosines des protéines, pour produire des 3-nitrotyrosines. Il peut également induire des lésions de I'ADN et la peroxydation des lipides. Dans le placenta, des résidus de 3-nitrotyrosine ont été observés dans des grossesses normales et prééclamptiques, principalement dans l'endothélium entourant le muscle lisse et le stroma villositaire. Dans la PE, I'une des cibles du peroxynitrite est la p38MAPK (p38 mitogen-activated protein kinase). Cette MAPK est nitratée dans les placentas PE [29], ce qui induit une baisse (de $65 \%$ ) de son activité catalytique (déclanchant ainsi la production de cytokines proinflammatoires, et le remodelage du tissu conjonctif).

\section{Endothélium vasculaire maternel et stress oxydant}

Les cellules ou facteurs circulants (neutrophiles activés, lipides peroxydés, xanthine oxydase, cytokines, etc.) peuvent agir sur les cellules endothéliales et déclencher un stress oxydant. Dans la PE, la production de $\mathrm{O}_{2}{ }^{--}$par les neutrophiles est augmentée. Plusieurs cytokines, dont le TNF- $\alpha$ (tumor necrosis factor alpha) retrouvé à des niveaux élevés dans le plasma de patientes $P E$, peuvent également générer un stress oxydant dans les cellules endothéliales, induisant ainsi des dommages directs et indirects via l'oxydation des LDL (low density lipoprotein) et l'activation de la voie $\mathrm{XO}$ et des NOX, principales sources de production de $\mathrm{O}_{2}{ }^{--}$dans les cellules endothéliales. 
Le radical N0*, qui provoque la relaxation du muscle lisse, régule aussi le tonus vasculaire, l'agrégation plaquettaire et l'adhérence des leucocytes. Il est synthétisé par les NO synthases (NOS) qui catalysent la conversion de la L-arginine en L-citrulline et oxyde nitrique. Les NOS génèrent également des anions $02^{*-}$ et du $\mathrm{H}_{2} \mathrm{O}_{2}$, en particulier si les concentrations intracellulaires de L-arginine ou de son cofacteur, le tétrahydrobioptérine (BH4), sont faibles. Des cellules HUVEC traitées avec $2 \%$ de plasma provenant de patientes prééclamptiques, montrent une expression et une activité accrues de l'arginase II, qui catabolise l'arginine en ornithine et en urée, ce qui réduit la disponibilité de L-arginine et augmente la production de $02^{--}$et de $0 \mathrm{NOO}^{-}$. L'inhibition de l'arginase, ou de la NOS endothéliale (eNOS), diminue la production de $02^{\circ-}$. L'arginase peut donc entraîner un découplage de l'eNOS, réduisant ainsi la production de $\mathrm{NO}^{\cdot}$ et favorisant la production de $02^{-}$. Les concentrations plasmatiques de L-arginine sont en fait diminuées chez les femmes présentant une $P \varepsilon$, alors que celles de diméthyle arginine asymétrique (ADMA), un inhibiteur de l'eNOS, sont augmentées. Ces modifications peuvent donc réduire la biodisponibilité du $\mathrm{NO}^{*}$ dans le système vasculaire maternel [30].

\section{Antioxydants et prééclampsie}

Le stress oxydant observé dans la PE peut résulter d'un défaut de disponibilité d'antioxydants. Les antioxydants sont classés en antioxydants enzymatiques: superoxyde dismutase (SOD), hémoxygénase (HO), catalase (CAT) et thiorédoxine (TRX); et antioxydants nonenzymatiques: glutathion (GSH), vitamines $C$ et $\varepsilon$, NADH et NADPH. La SOD catalyse la dismutation de I'O2-- en $\mathrm{O}_{2}$ et $\mathrm{H}_{2} \mathrm{O}_{2}$, ce dernier étant neutralisé par la CAT qui le décompose en $\mathrm{H}_{2} \mathrm{O}$ et $\mathrm{O}_{2}$. La TRX est une enzyme oxydoréductase. Elle facilite la réduction d'autres protéines par la formation de ponts disulfure entre cystéines. Le GSH neutralise les $\varepsilon R O$. II intervient également dans le maintien de la forme réduite des vitamines $C$ et $\varepsilon$. Le NADPH est, entre autres, impliqué dans la protection contre les $\varepsilon R O$ en régénérant le GSH.

Les grossesses prééclamptiques présentent des taux extrêmement variables d'antioxydants [31]. Les activités SOD et CAT, mesurées dans le placenta et le sérum des femmes atteintes de $P \varepsilon$, varient selon les études et l'expression de TRX est diminuée dans les placentas prééclamptiques, mais ses taux plasmatiques demeurent inchangés par rapport aux individus sains. Pour le GSH, des concentrations plasmatiques et placentaires plus faibles que celles mesurées chez les femmes présentant une grossesse normale ont été observées. II en est de même pour les taux plasmatiques de vitamine $C$ et $\varepsilon$ chez les femmes atteintes de $P E$.

\section{Modèles d'étude de la prééclampsie}

Les modèles animaux de prééclampsie :

points communs et différences

Chez l'animal, la PE peut être induite par des méthodes chirurgicales, pharmacologiques ou transgéniques [32]. Une importante observation, réalisée à partir de ces différents modèles, est que, bien que les voies affectées soient très différentes, les phénotypes résultants conjuguent tous une hypertension gestationnelle et une protéinurie, révélant l'aspect multifactoriel de la pathologie.

Les modèles de $P E$ ont été développés principalement chez les rongeurs (rat/souris), mais aussi les ovins et des primates cercopithécidés (babouins et macaques). L'un des plus anciens modèles utilisés dans plusieurs espèces, est le RUPP (restricted utero-placental perfusion). Cette opération chirurgicale réalisée au dernier tiers de la grossesse induit une $P \varepsilon$ tardive avec une augmentation d'expression de sFLTl. L'injection de sFLTl chez les rongeurs induit le syndrome prééclamptique. Néanmoins, il est vraisemblable que l'origine «naturelle » de la pathologie soit de nature immunologique, l'augmentation de sFLTl accompagnant la prééclampsie, avec une concentration souvent proportionnelle à la gravité de la maladie.

\section{Modèles cellulaires de la prééclampsie}

Au cours de la grossesse, les altérations liées à la $P E$ impactent les systèmes vasculaires maternels avec des effets pro-inflammatoires qui peuvent altérer la fonction placentaire. Plusieurs aspects clés de la PE sont étudiés in vitro, sur des cytotrophoblastes et des cellules endothéliales.

\section{Les cytotrophoblastes}

Des explants de villosités placentaires, des cytotrophoblastes primaires et des lignées cellulaires sont couramment utilisés afin d'étudier la fonction endocrine placentaire, le métabolisme et les mécanismes de transport, ainsi que pour disséquer les processus cellulaires impliqués (prolifération, différenciation, apoptose et fusion syncytiale) [33]. Les explants prélevés à partir de placentas de patientes atteintes de $P \varepsilon$ sont utilisés pour comparer leur état fonctionnel à ceux provenant de femmes à la grossesse non-pathologique. Cette approche présente cependant certaines limites: la disponibilité de matériel frais et l'absence de contrôle génétique des individus.

Une autre approche consiste en la réalisation de cultures primaires de cytotrophoblastes isolés à partir de villosités choriales (CTEV pour cytotrophoblastes extra-villeux). Elles permettent l'étude des mécanismes qui contrôlent l'invasion trophoblastique chez l'homme. Ces cellules conservent, in vitro, les caractéristiques des cytotrophoblastes in situ (expression de l'hormone placentaire lactogène, d'intégrines, de HLA-G, de Cytokératine-7, etc.) et permettent d'étudier in vitro l'invasion trophoblastique. Afin de les rendre immortelles, et donc de dépasser la limite de leur survie en culture, les cellules sont immortalisées par transfection 
des gènes $T / t$ du virus SV40 (polyomavirus simian virus 40). Des systèmes de coculture, en présence de cellules de myomètre ou de cellules endothéliales, permettent l'analyse des mécanismes impliqués dans les interactions entre les différents types cellulaires associés aux processus de placentations normale et pathologique. Des reconstructions du placenta en trois dimensions devraient également, à l'avenir, permettre d'élucider certains aspects de la pathologie. Des perfusions de placentas entiers sont, d'autre part, utilisées pour étudier le transfert placentaire de médicaments ou de perturbateurs endocriniens.

Des lignées cellulaires, dérivées de choriocarcinomes comme BeWo, JEG-3 et JAR, ont également été développées. BeWo est la plus utilisée comme substitut du trophoblaste villeux. Cette lignée présente la plupart des caractéristiques de ces cellules: expression des syncytines 1 et 2, sécrétion de hCG (hormone chorionique gonadotrope), de hPL (hormone lactogène placentaire), de progestérone et d'œstradiol. Sous l'action de la forskoline, qui active la voie de l'AMPc, les cellules de cette lignée fusionnent. Les BeWo permettent donc d'étudier la formation du syncytium trophoblastique, en hypoxie ou en présence de sérum provenant de patientes prééclamptiques [33], ou de déterminer les effets de molécules à potentiel thérapeutique (comme les vitamines $C$ et $\varepsilon$, l'aspirine et l'héparine). $\varepsilon$ n particulier, l'aspirine augmente la syncytialisation des cellules BeWo. Les vitamines $C$ et $\varepsilon$ n'ont pas d'effet significatif, mais après $72 \mathrm{~h}$ de traitement, elles déclenchent une augmentation de la sécrétion d'hCG. Les lignées dérivées de choriocarcinomes sont aussi particulièrement utiles pour étudier le rôle d'une cible d'intérêt dans la fonction trophoblastique normale et pathologique. Ainsi STOXI (storkhead box 1) qui code un facteur de transcription impliqué dans le développement de la $P E$, induit des modifications transcriptomiques mimant celles du placenta prééclamptique, en particulier des altérations de gènes cibles de l'hypoxie [34].

\section{Les cellules endothéliales}

Les altérations subies par l'endothélium maternel au cours de la PE ont fait l'objet d'études in vitro utilisant des cultures primaires ou de lignées immortalisées d'HUVEC. Plusieurs études ont concerné la réponse de ces cellules au plasma de femmes atteintes de PE [35]. Des facteurs, présents dans le plasma prééclamptique, augmentent la perméabilité, l'expression de la eNOS et de la NADPH oxidase, et la production de superoxyde et de peroxynitrite, induisant ainsi un stress. Des marqueurs de l'activation endothéliale comme la prostacycline, l'endothéline 1, le PDGF (platelet derived growth factor), VCAM-1 (vascular cell adhesion protein 1), ICAM-1 (intercellular adhesion molecule 1) sont aussi augmentés et une sécrétion accrue de chimiokines (MCP-1, monocyte chemotactic protein 1 ou CCL2 - chemokine ligand 2) et de cytokines (IL[interleukine]-6 et IL-8), a été observée. Le transcriptome des cellules HUVEC exposées au plasma prééclamptique montre l'induction de gènes codant des protéines impliquées dans la régulation du tonus vasculaire comme EDNI (endotheline 1), APLN (Apelin) et CBS (cystathionine beta-synthase) [36]. Finalement, des expériences de coculture de monocytes avec des cellules HUVEC ont montré que le plasma prééclamptique contient des facteurs qui activent les cellules monocytaires qui, à leur tour, stimulent les cellules endothéliales. Les médiateurs de ces interactions entre cellules restent cependant à identifier.

\section{Des possibilités thérapeutiques}

\section{L'enjeu majeur du diagnostic précoce}

Le seul traitement avéré de la PE est l'extraction du placenta; des manifestations postpartum peuvent néanmoins apparaître en raison des atteintes endothéliales maternelles. La première intervention est le contrôle de la pression artérielle maternelle par un traitement hypotenseur, de type bêtabloquant, comme le labétalol, ou de type inhibiteur de canaux calciques, et la prévention des atteintes neurologiques et des convulsions, avec le sulfate de magnésium. Le suivi médical consiste ensuite en une hospitalisation sous surveillance, dans un établissement associant structures de soins intensifs et unité de néonatologie adaptée à la prise en charge du nouveau-né, selon l'âge gestationnel et le développement staturo-pondéral (attitude d'expectative). La question du pronostic/diagnostic précoce pour une prévention de la $P \varepsilon$, avant ses manifestations cliniques, se pose afin de proposer une prise en charge adaptée et la mise en place d'un traitement prophylactique, comme de l'aspirine à faible dose. L'identification précoce des femmes enceintes susceptibles de développer une $P \varepsilon$ n'est pas possible. Des facteurs de risques de $P \varepsilon$ ont cependant été mis en évidence avec, en premier lieu un antécédent de PE (risque relatif [RR] de 8,4) et les conditions pré-conceptionnelles (classées par ordre décroissant de fréquence relative au sein de la population de femmes concernées [37]) : syndrome des antiphospholipides (17\%), hypertension artérielle chronique ( $16 \%, \mathrm{RR}: 5,1$ ), diabète ( $11 \%$, RR: 3,7 ), indice de masse corporelle (IMC) supérieur à 30 (7\%, $\mathrm{RR}: 2,8)$, et recours aux techniques de procréation assistée (6\%, RR: 1,8). Des algorithmes de prédiction ont été développés. Ils associent des caractéristiques maternelles et l'historique médical, des paramètres biophysiques et biochimiques, afin de réaliser des dépistages au premier trimestre (avant la 14-16 semaine d'aménorrhée). Une étude portant sur 60000 femmes, combinant des facteurs maternels, la pression artérielle moyenne, l'indice de pulsatilité de l'artère utérine, et le taux sérique du facteur de croissance du placenta (PIGF) a permis de détecter $76 \%$ des cas de PE précoce, et $38 \%$ des cas de PE tardive, avec un taux de faux négatifs de $10 \%$. La technicité nécessaire pour acquérir les paramètres biophysiques et biochimiques pour cet algorithme ne permet cependant pas, à ce jour, son implémentation pour un suivi de routine chez toutes les femmes enceintes. Une étude très récente a montré 


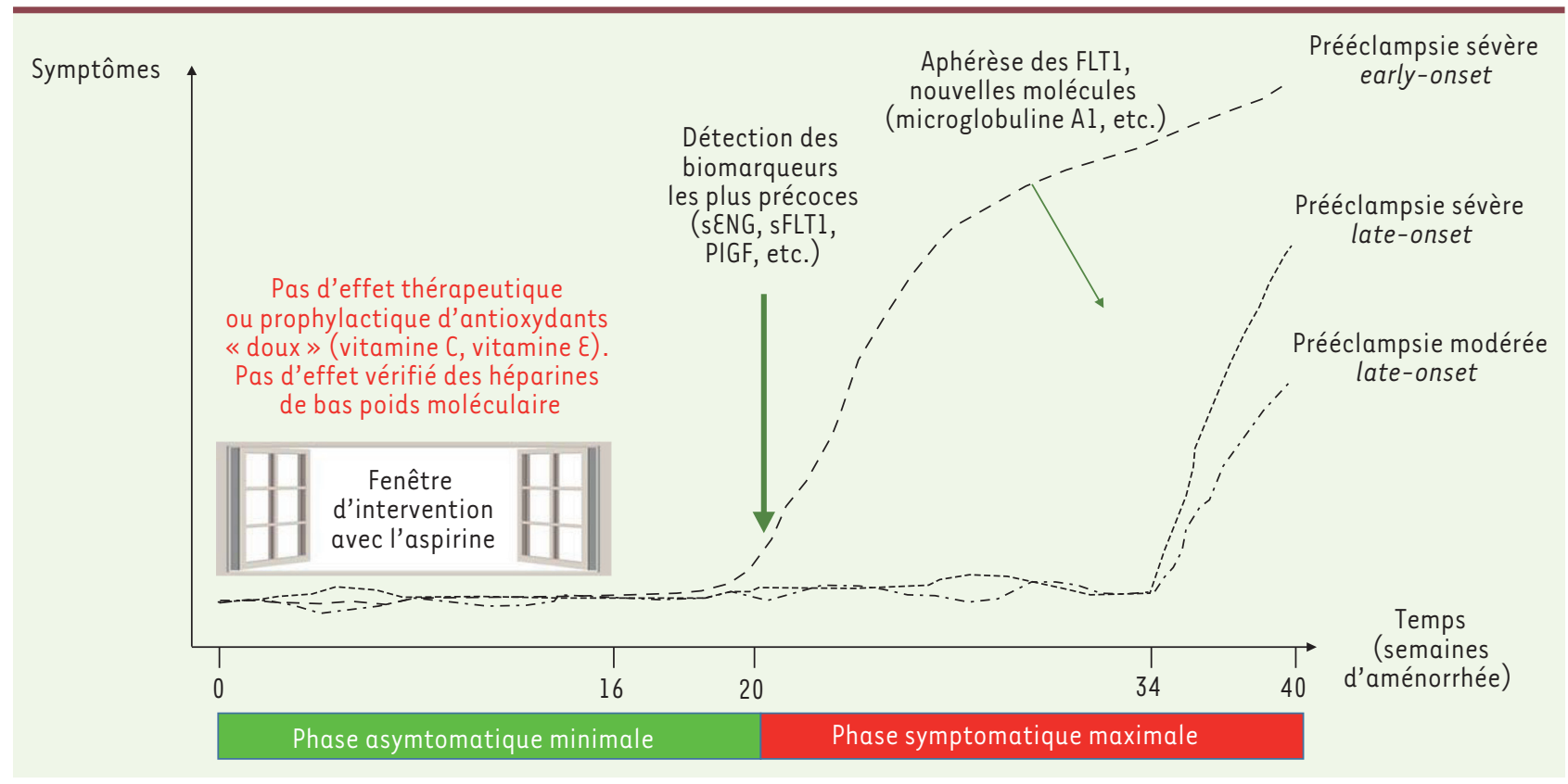

Figure 5. Une vision cinétique des possibilités d'action (limitées pour le moment) contre la prééclampsie. De nombreux auteurs distinguent des prééclampsies early-onset, en général plus graves, et late-onset plus fréquentes, qui peuvent parfois induire également des symptômes graves et tardifs, voire entraîner un décès maternel plusieurs jours après la sortie de la patiente de l’hôpital. De nombreuses études établissent un effet bénéfique de l'aspirine à faible dose à condition qu'elle soit administrée à des périodes précoces de la grossesse (avant la 16e semaine d’aménorrhée). Les modifications des marqueurs sériques les plus précoces ne sont détectables que vers la $20^{e}$ semaine d'aménorrhée, et donc trop tardivement pour agir. D'autres molécules (héparines, antioxydants par voie orale) n'ont pas montré d'efficacité contre la maladie. Des efforts de recherche de molécules susceptibles d'inverser les symptômes sont importants, avec la limite du rapport coût-bénéfice, toujours cruciale dans le domaine des thérapies possibles pendant la gestation. sENG : soluble endoglin; sFLTI : soluble fms related tyrosine kinase 1 ; PIGF : placental growth factor.

que ce genre d'algorithme peut être précieux pour décider de l'administration précoce présymptomatique d'aspirine à faible dose, dans le cadre du projet ASPRE (combined multimarker screening and randomized patient treatment with aspirin for evidence-based preeclampsia prevention) [38]. L'une des suites de ce projet a été l'étude de la population, analysée et traitée, sous l'angle d'hétérogénéités de sousgroupes, apparemment sans effet en fonction des caractéristiques maternelles ou de leur histoire obstétricale. L'effet de l'aspirine disparaissait cependant dans un contexte d'hypertension chronique [39]. Une étude de transcriptomique avait détecté une augmentation de sFTLl dans la PE et un taux accru est retrouvé dans le plasma des femmes présentant une $P \varepsilon$ précoce. Les concentrations de sENG et le ratio sFLTl/PIGF se sont également avérés être augmentés en cas de $P \varepsilon$. Les valeurs prédictives positives de ces mesures, seules, avant 20 semaines d'aménorrhée, restent cependant faibles alors que les valeurs prédictives négatives sont excellentes, ce qui permet de discriminer, dans une population à risque, les femmes qui ne développeront pas de $P E$ précoce [40]. Les outils actuels (paramètres cliniques, histoire médicale de la patiente, facteurs angiogéniques) sont utiles pour différencier les femmes présentant des symptômes de PE mais avec un profil angiogénique normal et un risque faible de complications liés à la $P E$ sévère. Ils permettraient de réduire des hospitalisations qui s'avèrent inutiles.

\section{Aspirine, héparines de bas poids moléculaire, et statines}

L'absence de traitement, autre que l'interruption de grossesse une fois que la maladie est symptomatique, et le concept d'une pathologie placentaire à l'origine d'une dysfonction vasculaire chez la mère, ont conduit à proposer des traitements anti-thrombotiques à titre préventif, comme l'aspirine, initialement en raison de ses effets anti-plaquettaires, des héparines de bas poids moléculaire, ou plus récemment des statines, chez des femmes à risque élevé.

Des méta-analyses d'essais cliniques randomisés (aspirine versus placebo ou versus absence de traitement) avaient suggéré que la prévalence de la $P E$ sévère, et du retard de croissance fœtal, pouvait être réduite par un traitement par l'aspirine, initié avant 16 semaines d'aménorrhée (SA). L'étude ASPRE a comparé une dose de $150 \mathrm{mg}$ par jour d'aspirine à un placebo, de la 11-14 SA jusqu'à 36 SA, chez des femmes qui avaient été dépistées pour avoir un risque élevé de PE précoce, selon l'algorithme alliant paramètres cliniques, mesures biophysiques/biochimiques [38]. Une diminution significative de la survenue de $P E$ précoce dans le groupe 
« aspirine » a été observée (13 PE précoces sur 798 participantes $1,6 \%$ - dans le groupe aspirine, comparées à 35 sur $822-4,3 \%$-dans le groupe placebo, avec un risque relatif ajusté exprimé en odds ratio dans le groupe aspirine : 0,38; intervalle de confiance à $95 \%: 0,20$ to $0,74 ; P=0,004)$. Le dépistage de 26000 femmes enceintes d'un singleton (c'est-à-dire une grossesse non gémellaire) a été nécessaire pour obtenir une puissance suffisante, avec, comme résultat, 20 cas de PE précoces évités. Les résultats obtenus démontrent ainsi l'effet positif de l'aspirine dans les cas de PE précoce, conformément à plusieurs méta-analyses antérieures soulignant la nécessité d'administrer l'aspirine avant 16 semaines de grossesse.

Une méta-analyse récente de huit essais cliniques randomisés réalisés entre 2000 et 2013 testant la capacité d'un traitement prospectif par héparines de bas poids moléculaire, pour la prévention, entre autres, d'une PE précoce n'a pas montré d'effet significatif [41]. Pour les statines, quelques études pilotes, restreintes en effectif, suggèrent un intérêt en clinique possible pour la pravastine [42]. Les résultats d'études cliniques de plus grande ampleur seront nécessaires avant de proposer ces nouveaux traitements.

\section{Nouvelles drogues, nouveaux modes d'application}

La recherche thérapeutique en PE implique l'utilisation de nouvelles médications. Cependant, aucune n'a vraiment, pour le moment, pu montrer son efficacité, en raison de la difficulté d'utiliser des médicaments pendant la grossesse (Figure 5). Néanmoins, certaines molécules sont en cours d'évaluation comme l'alpha-1-microglobuline, un chélateur naturel du fer [43] qui pourrait être administré aux femmes à risque. Parmi les autres molécules utilisables qui pourraient réduire la quantité de sFLTl et de sENG, mentionnons la metformine [44]. Une autre piste pourrait être fournie par l'administration de nanoparticules ciblant le trophoblaste [45], qui pourrait véhiculer des siARN (en anglais, small interfering RNA) mimant l'action des miARN naturels. Récemment, il a été proposé de limiter la circulation de sFLTl pour permettre une prolongation de la grossesse dans des conditions satisfaisantes de sécurité pour la mère et le fœtus [46]. Jusqu'à présent, ces études ont concerné très peu de patientes. Les bénéfices sont relativement modérés et la technique ne peut être implémentée à une grande échelle, suggérant que cette approche est plus intéressante en termes de recherche et de compréhension des bases moléculaires de la $P \varepsilon$ que d'utilisation clinique. $\diamond$

\section{SUMMARY}

New perspectives on preeclampsia

Preeclampsia is a major, frequent and potentially severe condition of pregnancy, characterized by severe hypertension and proteinuria. In this review, we describe recent advances in understanding the pathology, and discuss the long-term impacts on maternal vascular health. Next, we describe the genetic, epigenetic and immunological basis of preeclampsia. We describe the links between preeclampsia and oxidative stress in placental (trophoblast) and endothelial cells. We mention cellular and animal models commonly used to decipher modified pathophysiological pathways in a preeclamptic pregnancy compared to a normal pregnancy. Finally, we discuss the therapeutic options, readily available or in development, to improve the monitoring of pregnancies, the health of patients and that of children born from preeclamptic pregnancies. $\diamond$

\section{LIENS D'INTÉRÊT}

Les auteurs déclarent n'avoir aucun lien d'intérêt concernant les données publiées dans cet article.

\section{RÉFÉRENCES}

1. Rigourd V, Chelbi ST, Vaiman D. La pré-éclampsie. Med Sci (Paris) 2008 ; 24 : 1017-9.

2. Robillard Py, Scioscia M, Coppola D, et al. La «Donna di Ostuni », a case of eclampsia 28,000 years ago? J Matern Fetal Neonatal Med 2017 : 1-4.

3. Powe CE, Levine RJ, Karumanchi SA. Preeclampsia, a disease of the maternal endothelium: the role of antiangiogenic factors and implications for later cardiovascular disease. Circulation $2011 ; 123: 2856-69$.

4. Sergent F, Hoffmann P, Brouillet $S$, et al. Sustained endocrine gland-derived vascular endothelial growth factor levels beyond the first trimester of pregnancy display phenotypic and functional changes associated with the pathogenesis of pregnancy-induced hypertension. Hypertension 2016; 68 : 148-56.

5. Vikse BE, Irgens LM, Leivestad T, et al. Preeclampsia and the risk of endstage renal disease. $N$ Engl J Med 2008 ; $359: 800-9$.

6. Timpka S, Macdonald-Wallis C, Hughes AD, et al. Hypertensive disorders of pregnancy and offspring cardiac structure and function in adolescence. J Am Heart Assoc 2016; 5

7. Buffat C, Mondon F, Rigourd V, et al. A hierarchical analysis of transcriptome alterations in intrauterine growth restriction (IUGR) reveals common pathophysiological pathways in mammals. J Pathol 2007 ; 213 : 337-46.

8. Amaral LM, Cunningham MW Jr., Cornelius DC, LaMarca B. Preeclampsia: long-term consequences for vascular health. Vasc Health Risk Manag 2015 ; $11: 403-15$.

9. van Kesteren F, Visser S, Hermes W, et al. Prevention of cardiovascular risk in women who had hypertension during pregnancy after 36 weeks gestation. Hypertens Pregnancy $2015 ; 34: 261-9$.

10. Mongraw-Chaffin ML, Cirillo PM, Cohn BA. Preeclampsia and cardiovascular disease death: prospective evidence from the child health and development studies cohort. Hypertension $2010 ; 56: 166-71$.

11. Cnattingius S, Reilly M, Pawitan Y, Lichtenstein P. Maternal and fetal genetic factors account for most of familial aggregation of preeclampsia: a population-based Swedish cohort study. Am J Med Genet A 2004 ; 130A : 365-71.

12. Staines-Urias $\varepsilon$, Paez MC, Doyle P, et al. Genetic association studies in preeclampsia: systematic meta-analyses and field synopsis. Int J Epidemiol $2012 ; 41: 1764-75$.

13. McGinnis R, Steinthorsdottir V, Williams NO, et al. Variants in the fetal genome near FLTl are associated with risk of preeclampsia. Nat Genet 2017 ; 49 : 1255-60.

14. Chelbi ST, Wilson ML, Veillard AC, et al. Genetic and epigenetic mechanisms collaborate to control SERPINA3 expression and its association with placental diseases. Hum Mol Genet 2012 ; 21 : 1968-78.

15. Vaiman D. Genes, epigenetics and miRNA regulation in the placenta. Placenta $2017 ; 52$ : $127-33$

16. Doridot L, Miralles F, Barbaux S, Vaiman D. Trophoblasts, invasion, and microRNA. Front Genet $2013 ; 4: 248$.

17. Lagana AS, Vitale SG, Sapia F, et al. miRNA expression for early diagnosis of preeclampsia onset: hope or hype? J Matern Fetal Neonatal Med $2017: 1-5$.

18. LaMarca B, Cornelius D, Wallace K. Elucidating immune mechanisms causing hypertension during pregnancy. Physiology (Bethesda) $2013 ; 28: 225-33$.

19. Robillard Py, Hulsey TC. Association of pregnancy-induced-hypertension, pre-eclampsia, and eclampsia with duration of sexual cohabitation before conception. Lancet $1996 ; 347: 619$.

20. Hiby SE, Apps R, Sharkey AM, et al. Maternal activating KIRs protect against human reproductive failure mediated by fetal HLA-C2. J Clin Invest 2010 ; $120: 4102-10$. 


\section{RÉFÉRENCES}

21. Schwede S, Alfer J, von Rango U. Differences in regulatory T-cell and dendritic cell pattern in decidual tissue of placenta accreta/increta cases. Placenta 2014 ; 35 : 378-85.

22. Kalyanaraman $B$. Teaching the basics of redox biology to medical and graduate students: Oxidants, antioxidants and disease mechanisms. Redox Biol 2013 ; 1 : 244-57.

23. Elliot MG. Oxidative stress and the evolutionary origins of preeclampsia. J Reprod Immunol 2016 ; $114: 75-80$.

24. Challier JC, Uzan S. Le placenta humain et ses pathologies : I'oxygène en question. Med Sci (Paris) $2003 ; 19: 1111-20$.

25. Shi Z, Long $\mathrm{W}$, Zhao $\mathrm{C}$, et al. Comparative proteomics analysis suggests that placental mitochondria are involved in the development of pre-eclampsia. PLoS One $2013 ; 8$ : e64351.

26. Many A, Hubel CA, Fisher SJ, et al. Invasive cytotrophoblasts manifest evidence of oxidative stress in preeclampsia. Am J Pathol $2000 ; 156: 321-31$.

27. Matsubara K, Matsubara $Y$, Hyodo S, et al. Role of nitric oxide and reactive oxygen species in the pathogenesis of preeclampsia. J Obstet Gynaecol Res 2010 ; $36: 239-47$.

28. Dechend R, Viedt C, Muller DN, et al. ATl receptor agonistic antibodies from preeclamptic patients stimulate NADPH oxidase. Circulation 2003; 107 : 1632-9.

29. Myatt L. Review: Reactive oxygen and nitrogen species and functional adaptation of the placenta. Placenta 2010 ; 31 (suppl) : S66-9.

30. Sanchez-Aranguren LC, Prada C $\varepsilon$, Riano-Medina CE, Lopez M. Endothelial dysfunction and preeclampsia: role of oxidative stress. Front Physiol $2014 ; 5: 372$

31. Bilodeau JF. Review: maternal and placental antioxidant response to preeclampsia-impact on vasoactive eicosanoids. Placenta $2014 ; 35$ (suppl) : S32-8.

32. Erlandsson L, Naav A, Hennessy A, et al. Inventory of Novel Animal Models Addressing Etiology of Preeclampsia in the Development of New Therapeutic/Intervention Opportunities. Am J Reprod Immunol $2016 ; 75: 402-10$.

33. Orendi K, Gauster M, Moser G, et al. Effects of vitamins $C$ and $\varepsilon$, acetylsalicylic acid and heparin on fusion, beta-hCG and PP13 expression in BeWo cells. Placenta $2010 ; 31: 431-8$.

34. Rigourd V, Chauvet C, Chelbi ST, et al. STOXI overexpression in choriocarcinoma cells mimics transcriptional alterations observed in preeclamptic placentas. PLoS One 2008; 3 : e3905.

35. Goulopoulou S, Davidge ST. Molecular mechanisms of maternal vascular dysfunction in preeclampsia. Trends Mol Med 2015 ; 21 : 88-97.

36. Calicchio R, Buffat C, Mathieu JR, et al. Preeclamptic plasma induces transcription modifications involving the AP-1 transcriptional regulator JDP2 in endothelial cells. Am J Pathol 2013; 183 : 1993-2006.

37. Bartsch $\varepsilon$, Medcalf KE, Park AL, Ray JG. Clinical risk factors for pre-eclampsia determined in early pregnancy: systematic review and meta-analysis of large cohort studies. BMJ $2016 ; 353$ : il 1753.
38. Rolnik DL, Wright D, Poon LC, et al. Aspirin versus placebo in pregnancies at high risk for preterm preeclampsia. $N$ Engl J Med 2017 ; 377 : 613-22.

39. Poon LC, Wright D, Rolnik DL, et al. Aspirin for evidence-based preeclampsia prevention trial: effect of aspirin in prevention of preterm preeclampsia in subgroups of women according to their characteristics and medical and obstetrical history. Am J Obstet Gynecol 2017 ; pii : S0002-9378(17)30929-8. doi: 10.1016/j.ajog.2017.07.038.

40. Zeisler H, Llurba $\varepsilon$, Chantraine F, et al. Predictive Value of the sFlt-1:PIGF Ratio in Women with Suspected Preeclampsia. N EnglJ Med 2016 ; 374 : 13-22.

41. Rodger MA, Gris JC, de Vries JIP, et al. Low-molecular-weight heparin and recurrent placenta-mediated pregnancy complications: a meta-analysis of individual patient data from randomised controlled trials. Lancet 2016; $388: 2629-41$.

42. Lefkou $\varepsilon$, Mamopoulos A, Dagklis T, et al. Pravastatin improves pregnancy outcomes in obstetric antiphospholipid syndrome refractory to antithrombotic therapy. J Clin invest 2016; 126 : 2933-40.

43. Gram M, Anderson UD, Johansson ME, et al. The human endogenous protection system against cell-free hemoglobin and heme is overwhelmed in preeclampsia and provides potential biomarkers and clinical indicators. PLoS One 2015 ; 10 : e0138111.

44. Brownfoot FC, Hastie R, Hannan NJ, et al. Metformin as a prevention and treatment for preeclampsia: effects on soluble fms-like tyrosine kinase 1 and soluble endoglin secretion and endothelial dysfunction. Am J Obstet Gynecol $2016 ; 214: 356$ el- 15.

45. King A, Ndifon C, Lui S, et al. Tumor-homing peptides as tools for targeted delivery of payloads to the placenta. Sci Adv 2016 ; 2 : el600349.

46. Thadhani R, Kisner T, Hagmann $\mathrm{H}$, et al. Pilot study of extracorporeal removal of soluble fms-like tyrosine kinase 1 in preeclampsia. Circulation $2011 ; 124: 940-50$

47. Brouillet $S$, Hoffmann P, Alfaidy N, Feige JJ. Prokinéticines. De nouveaux peptides régulateurs de la reproduction humaine. Med Sci (Paris) $2014 ; 30$ : $274-9$

TIRÉS À PART

D. Vaiman

u'est-ce que la guérison? Des réponses, il y en a. De toutes sortes et de tout temps. Chacun y va de son savoir, religieux, scientifique, médical... Et de quoi est-on supposé guérir ? D’un symptôme, d'une douleur, d'une maladie, d'une répétition mortifère, d'un destin mélancolique? Pour la psychanalyse, la guérison s'insère dans un système imaginaire et a, comme point de mire, un idéal. «La guérison, c'est une demande... » précise Lacan. Les auteurs nous invitent ici à découvrir, au-delà du semblant et à

Qu'est-ce que la guérison pour la psychanalyse?
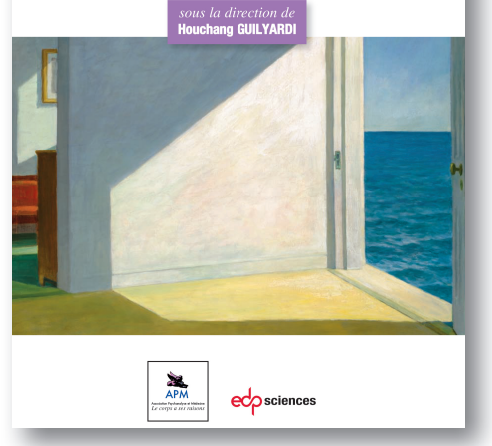

ISBN : 978-2-7598-1864-8 322 pages $24 €$ partir de la clinique, les liens entre guérison et vérité du sujet.

Comité éditorial de l'Association Psychanalyse et Médecine (APM) : Martine Dombrosky, Sophie Dunoyer de Segonzac, Houchang Guilyardi, Josette Olier, Betty Testud

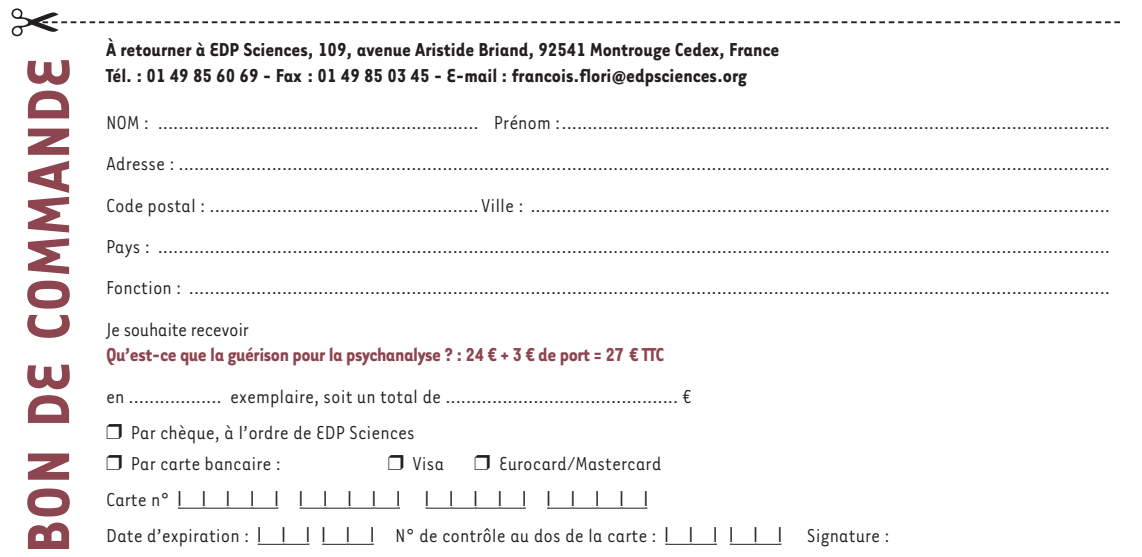

